\title{
Ultrasound suprainguinal fascia iliaca block in knee surgery
}

\author{
Rodrigo Sanllorente-Sebastián ${ }^{1} \cdot$ Begoña Arroyo-García ${ }^{1} \cdot$ Rodrigo Avello-Taboada $^{1}$
}

Received: 28 January 2020 / Accepted: 22 February 2020 / Published online: 20 March 2020

(c) Japanese Society of Anesthesiologists 2020

To the Editor:

We read with great interest the article from Vermeylen about suprainguinal fascia iliaca compartment block (SFICB) [1] .

We want to share our experience performing SFICB for postoperative analgesia in total knee arthroplasty (TKA).

Ultrasound-guided FICB is a fascial block, with two possible approaches, infrainguinal or suprainguinal, aiming femorocutaneous, femoral and obturator nerves, all together in a single-shot technique [2]. Nevertheless, suprainguinal approach to FICB leads to a more consistent block of the three target nerves [1,2].

We routinely perform postoperative nerve blocks with analgesic purposes for TKA in the Postanesthesia Care Unit (PACU), as there is not enough time to perform them in the operating room.

Ultrasound probe is placed in a sagittal oblique plane at anterosuperior iliac spine pointing to the umbilicus. Then it is moved inferomedially along the inguinal ligament until the hourglass sign is observed, formed by the sartorius, abdominal and iliac muscles (Supplementary Fig. 1). Then a 70-mm echogenic needle (AKUS, Murcia, Spain) is inserted in plane in a caudal to cranial fashion until the fascia iliaca is reached. Then $40 \mathrm{ml}$ of ropivacaine $0.25 \%$ plus $4 \mathrm{mg}$ dexamethasone are injected. All patients also received paracetamol and NSAIDs routinely for a multimodal approach.

In 13 TKA patients, the pain was reduced, although three patients needed $2 \mathrm{mg}$, one needed $3 \mathrm{mg}$ and two needed $4 \mathrm{mg}$ intravenous morphine. In the ward, in the next $24 \mathrm{~h}$, two patients did not have good analgesic control.

Further research is needed.

\section{References}

1. Vermeylen K, Soetens F, Leunen I, Hadzic A, Van Boxtael S, Pomés J, et al. The effect of the volume of supra-inguinal injected solution on the spread of the injectate under the fascia iliaca: a preliminary study. J Anesth. 2018;32(6):908-13.

2. Desmet M, Balocco AL, Van Belleghem V. Fascia iliaca compartment blocks: different techniques and review of the literature. Best Pract Res Clin Anaesthesiol. 2019;33(1):57-66.

Publisher's Note Springer Nature remains neutral with regard to jurisdictional claims in published maps and institutional affiliations.

Electronic supplementary material The online version of this article (https://doi.org/10.1007/s00540-020-02754-2) contains supplementary material, which is available to authorized users.

This comment refers to the article available online at https://doi. org/10.1007/s00540-018-2558-9.

Rodrigo Sanllorente-Sebastián

bibliotecas2010@gmail.com

1 Hospital Universitario de Burgos, Burgos, Spain 